\title{
Automated Model-Based Rib Cage Segmentation and Labeling in CT Images
}

\author{
Tobias Klinder ${ }^{1,2}$, Cristian Lorenz ${ }^{2}$, Jens von Berg ${ }^{2}$, Sebastian P.M. Dries ${ }^{2}$, \\ Thomas Bülow ${ }^{2}$, and Jörn Ostermann ${ }^{1}$ \\ ${ }^{1}$ Institut für Informationsverarbeitung, University of Hannover, Germany \\ klinder@tnt.uni-hannover.de \\ ${ }^{2}$ Philips Research Europe - Hamburg, Sector Medical Imaging Systems, Germany*
}

\begin{abstract}
We present a new model-based approach for an automated labeling and segmentation of the rib cage in chest CT scans. A mean rib cage model including a complete vertebral column is created out of 29 data sets. We developed a ray search based procedure for rib cage detection and initial model pose. After positioning the model, it was adapted to 18 unseen CT data. In 16 out of 18 data sets, detection, labeling, and segmentation succeeded with a mean segmentation error of less than $1.3 \mathrm{~mm}$ between true and detected object surface. In one case the rib cage detection failed, in another case the automated labeling.
\end{abstract}

\section{Introduction}

Although bony structures show high contrast in Computed Tomography (CT) images, their detection, identification, and correct segmentation remain still a challenge today. The image analysis process is complicated by, e.g., similarity of adjacent structures to the object to be segmented, partial volume effects resulting in no clear object boundary, or the fact that scans frequently contain pathology. One attempt to overcome these difficulties is to include prior knowledge in the form of anatomical models.

The rib cage shapes the human chest protecting all inner soft-tissue organs. For that reason, a geometric model of the osseous thorax is of special interest because it can serve as a reference for the location of soft-tissue organs. A model of the vertebral column is in itself of clinical relevance since it may support orthopedic and neurological applications. In this context, a precise segmentation of the vertebral column including an identification of the individual vertebrae is essential.

Model-based segmentation is known to be dependent on a good initialization. Once adaptation is misled, it can hardly recover. Especially in the case of the rib cage with its amount of similar adjacent structures, careful positioning is an important point.

\footnotetext{
* We would like to thank Katrina Read from Philips Medical Systems, Cleveland (USA) as well as the University of Maryland Medical Center, Baltimore (USA) for all image data.
} 
To our knowledge not many publications address the segmentation and labeling of the rib cage or the complete vertebral column. In contrast to semiautomatic region-based approaches, Shen et al. present a fully automatic tracingbased algorithm extracting and identifying rib centerlines 11. A different work also providing a segmentation and labeling of individual ribs is done by Staal et al. 2]. Compared to our method, these approaches are specifically tuned to ribs and do not offer an additional vertebra segmentation.

From the modeling perspective, some work has been done in modeling particular complex anatomical objects as, e.g., the human heart [3]. Also the modeling of individual vertebrae has been addressed earlier [4. However, to our knowledge it is the first time that a complete osseous thorax has been modeled and adapted to $\mathrm{CT}$ data scans. With its amount of particular structures, the thorax is thereby an extension from individual object to object-group modeling.

The remainder of this text is organized as follows. Section 2.1] describes the setup of the initial rib cage model. For positioning of the complete model to given CT data, a ray search based approach was developed which is introduced in Sect. 2.2. As an alternative, Sect. 2.3 describes an iterative positioning of vertebra models. By using these approaches, relevant structures were segmented in 29 CT data sets from which a mean model has been built as described in Sect. 2.4. The results of the positioning and adaptation of the mean model to unseen data are given in Sect. 3 .

\section{Methods}

\subsection{Inital Model Setup}

Triangulated surface models of all 24 ribs and all 24 presacral vertebrae were initially created. Vertebra model generation was based on the scanning of commercially available plastic phantoms with a Philips Brilliance40 CT scanner. For rib model generation, an interactive segmentation from patient CT data was performed similar to [3. By adapting all created surface models to their corresponding anatomical objects in reference patient CT data, the individual models were assembled to form an initial rib cage model including a complete vertebral column on the basis of patient data. Adaptation was done using a shape-constrained deformable surface model approach 4, which minimizes an energy term consisting of internal $E_{\text {int }}$ (shape similarity) and external $E_{\text {ext }}$ (image features) energies.

Due to the characteristic shape of the ribs, we establish a centerline-based description as an alternative for rib surface meshes. We calculate a rib's centerline by iteratively cutting a given rib mesh with planes being perpendicular to the surface. The center points of the obtained cut contours correspond to the desired centerline points. Shape information is added to the centerline by calculating an ellipse fit [5] to each cut contour. Between centerline points and ellipse parameters, we interpolate using B-Splines. Compared to a simple surface mesh description, this alternative enables an automatic rib surface generation when the location of the centerline of a rib is given and the centerline is identified. 


\subsection{Global Model Positioning}

Before positioning the model to a data set, at least parts of the corresponding structures have to be found. Since the ribs provide a framework for the entire chest, a proper positioning for the complete model can be determined from a correct rib detection. For object detection, we apply a ray search based approach 6]. By sending rays through the data set searching for a typical gray value profile, rib candidates can be detected. Due to the characteristic shape of the thorax, rib detection is divided into two symmetrical problems - one search for the left and one for the right ribs. In each case, a radial cylindrical ray search is applied in every image slice with an angular sampling of $n=180$ and the cylinder axes pointing in head-foot direction. The location of the cylinder axes are determined by the top points of the lungs.

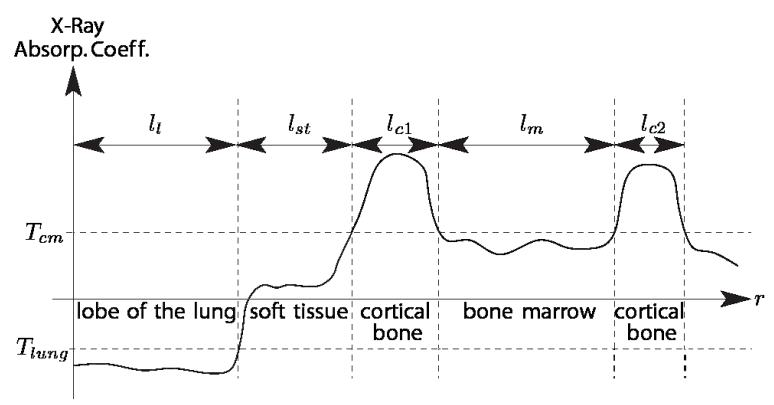

Fig. 1. Defined search profile for rib detection. The individual lengths are able to vary between zero and empirically determined maximal values. The thresholds $T_{\mathrm{cm}}$ and $T_{\text {lung }}$ separate the particular crossings. While $T_{\text {lung }}$ is constantly set to $T_{\text {lung }}=-100 \mathrm{HU}$, the exact value for $T_{\mathrm{cm}}$ has to be automatically determined for each patient.

With the settings of the cylindrical ray searches, the profile for rib detection can be defined. A ray crossing a rib bone shows a pattern of a high gray value when entering the rib through cortical bone followed by a lower one when traversing the bone marrow and again a high gray value exiting the rib through cortical bone. Before crossing a rib, a ray passes through lung tissue and a certain length of soft tissue. The entire profile is shown in Fig. 1 If a ray owns the defined profile, the middle point of the two positions, where the ray enters and exits the rib, is saved as a rib candidate. After detection, left and right rib centerlines are extracted seperately out of the candidates by using the coordinate system of the radial cylindrical ray searches as a reference. At first, the center of mass of candidates of successive slices obtained from rays with the same angle in the cylinder coordinate system is calculated. Afterwards, all combined candidates are grouped to individual rib clusters. Neighbouring candidates belong to the same cluster if they have approximately the same distance to the origin of the cylinder coordinate system and do not significantly vary in their z-coodinate. 
Model centerlines and extracted centerlines are registered with an iterative closest point (ICP) algorithm [7] allowing an affine transformation. The extracted centerlines are identified by registering all possible model combinations. If for instance seven rib pairs are extracted, combination 1-7, 2-8,..6-12 are registered. The configuration with the minimal residual error is supposed to correspond to the true configuration. After identifying the detected ribs and iteratively registering model centerlines to candidates, we apply a thin-plate spline approximation [8] of centerline points in order to cope with inter-patient variability of rib centerlines and to provide an improved positioning for the following segmentation. To take possible outliers into account, approximation is preferred to pure interpolation. An example for the registration is given in Fig. 2 .

With the found location of the rib centerlines and their identification, rib surface models are automatically generated as mentioned in Sect.2.1. The initial positioning of the vertebrae is given by applying the transformation obtained from the rib cage model registration. However, the vertebrae can be segmented more precisely when translating the vertebra models in direction of the mean difference vectors calculated out of the locations of the first centerline points of corresponding ribs after global model registration and thin-plate spline approximation.
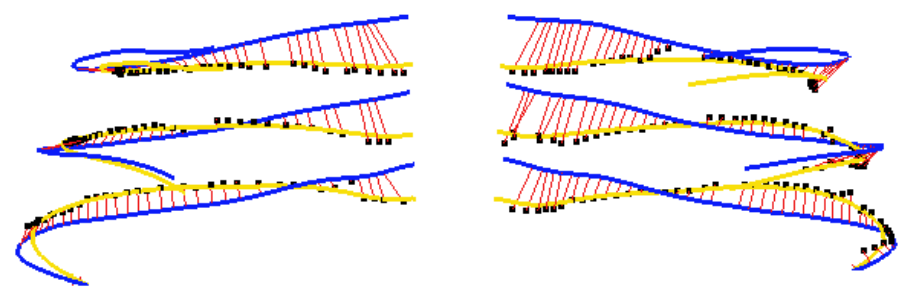

Fig. 2. An example for the entire registration process. The black dots correspond with the extracted centerlines of the rib candidates. The blue lines are the registered centerlines of the model using ICP. The red lines indicate the difference vectors of candidates and closest model centerline point of the corresponding rib. After thinplate spline approximation, the final positioning of the model is found (yellow). See electronic version for color figure.

\subsection{Iterative Vertebra Model Positioning}

Global model positioning requires thoracic CT scans covering the entire chest. Since the approach determines the initial model pose by detecting ribs it can not be applied for, e.g., lumbar or head-neck scans. Due to this fact, we developed an alternative model positioning for the vertebra models based on object relations. In order to express relations between the individual vertebrae, we have defined a local vertebra coordinate system (VCS). Since the shape of the vertebrae significantly changes down the spine, the VCS had to be derived from typical invariant object characteristics.

The definition of the VCS is based on the automatic calculation of three object-related simplified representations: a cylinder fit to the vertebral foramen, 
the middle plane of the upper and lower vertebral body surfaces, and the vertebra's sagittal symmetry plane (see Fig. 3). Out of the three representations, the VCS was defined. The intersection point of the axis of the fitted cylinder with the middle plane defines the origin of the VCS. The normal vector of the middle plane defines the $\mathbf{z}_{\mathrm{vcs}}$-axis. The $\mathbf{x}_{\mathrm{vcs}}$-axis is defined as the orthogonal component of the normal vector of the symmetry plane to the $\mathbf{z}_{\mathrm{vcs}}$-axis. The $\mathbf{y}_{\mathrm{vcs}}$-axis is defined as the cross-product of the $\mathbf{z}_{\mathrm{vcs}}$ - and the $\mathbf{x}_{\mathrm{vcs}}$-axis.

By using the derived object relations expressed in the form of VCSs, the vertebra models can be iteratively positioned. Starting from one adapted vertebra model, neighboring models can be positioned in the data set by applying the transformation between corresponding vertebrae obtained from the vertebral column model. From this initial position, the models are automatically adapted using again [4] and their patient specific VCSs are calculated. An iterative repetition of this process provides a segmentation of the shown extract of the vertebral column.

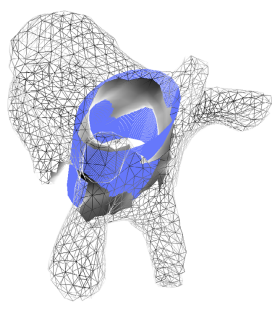

(a) Cylinder fit



(b) Middle plane

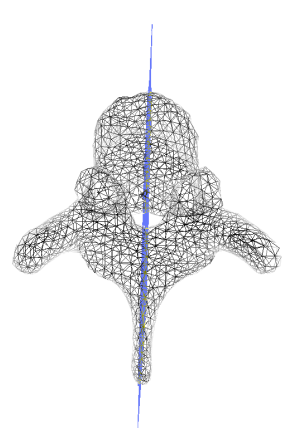

(c) Symmetry plane

Fig. 3. Different vertebra representations. An example for a cylinder fit to the vertebral foramen is given in (a). Figure (b) shows the middle plane of the vertebral body upper and lower surfaces. In each case, the corresponding automatically detected triangles are shown as filled. The symmetry plane is shown in (c).

\subsection{Mean Model Building}

For mean model generation, we adapted the initial model to a sample of $29 \mathrm{CT}$ data sets showing different portions of the rib cage and the vertebral column. In 17 data sets, all being chest CT scans, the model was positioned with the approach described in Sect. 2.2 and adapted using [4. In the other twelve data sets, being whole spine, head-neck, thoracic, and lumbar scans, only the vertebrae were segmented using the iterative positioning from Sect. 2.3. In each case, the automatic adaptation was inspected by the first author and local misadaptations were manually corrected. A clinical expert verified the results. By adapting the same particular surface models to different patient data sets, point correspondences between the models were preserved.

Usually, mean model building of individual objects is based on initially registering all corresponding meshes to one reference by a single global transformation 
and then averaging all vertex positions. However, in the case of a constellation of objects as, e.g., the osseous thorax not only the mean shape of the individual objects but also their mean location relative to the others has to be found. For that reason, we apply an iterative registration and averaging process. We start with the selection of one reference vertebra, register all corresponding vertebrae from other samples to that chosen reference using a rigid transformation (6 degrees of freedom) and finally average vertex positions. Starting from the calculated mean vertebra model, we register corresponding vertebrae to the mean shape and apply the transformation at first on the upper neighboring vertebrae. By averaging again the vertex positions of the transformed neighboring vertebrae, we obtain the corresponding mean shapes and also their mean location relative to the start mean vertebra model. An iterative execution of this process provides the upper part of the mean vertebral column. In the same manner, the lower part can be obtained. One advantage of this procedure is the ability to cope with sample data containing different portions of the vertebral column. In the case of the ribs, corresponding neighboring vertebrae are registered with the mean vertebra models. The obtained transformation is then applied on the rib models and finally vertex positions are again averaged.

\section{Results}

For adaptation of the mean rib cage model to unseen data, we followed the two different approaches for model positioning from Sect. 2.2 and Sect. 2.3. Both approaches were performed on 18 chest CT data sets that were part of the ensemble of the 29 data sets. In order to simulate model adaption to unseen data, the mean rib cage model has been generated by leaving out in each case the data set under consideration. All 18 data sets show a resolution of $0.85-0.97$ $\mathrm{mm}$ in $\mathbf{x}$ - and $\mathbf{y}$-direction and $2.5 \mathrm{~mm}$ in $\mathbf{z}$-direction.

The results of the automatic adaption were inspected by the first author and manually corrected if necessary. Again, a physician verified the corrections. In each case, the mean and maximal distances between adapted and corrected rib and vertebra models were calculated for all meshes of an entire data set and afterwards averaged resulting in mean and maximal distance values, $d_{\text {mean }}$ and $d_{\max }$. As distance measure, we calculated the Euclidian distance between corresponding vertices.

Automatic rib detection and subsequent extraction of rib centerlines following Sect. 2.2 was successful in 17 data sets. In the remaining case, the ray search based approach could only find very few candidates so that the centerline extraction failed and the rib cage model could not be positioned. In 16 out of the remaining 17 cases the following identification succeeded. One data set did not show a significant minimum after ICP registration. After finding an initial position, all particular surface models were adapted individually to their corresponding anatomical structure using [4]. The complete procedure including detection, identification, positioning, and adaptation took less than 5 min on a workstation with $2.16 \mathrm{GHz}$. The results of the segmentation are shown in Table 1 . With a 
Table 1. Summary of model adaptation to 16 data sets

\begin{tabular}{c||c|c|c||c|c|c}
\hline \multicolumn{1}{c||}{} & \multicolumn{3}{c||}{ vertebra adaptation } & \multicolumn{3}{c}{ rib adaptation } \\
& min & mean & max & min & mean & max \\
\hline \hline$d_{\text {mean }}[\mathrm{mm}]$ & 0.30 & 1.27 & 1.8 & 0.21 & 0.36 & 0.92 \\
$d_{\text {max }}[\mathrm{mm}]$ & 4.35 & 6.27 & 9.41 & 3.31 & 7.01 & 11.43 \\
\hline
\end{tabular}

mean distance error over all data sets of $1.27 \mathrm{~mm}$ for the vertebrae and $0.36 \mathrm{~mm}$ for the ribs, we achieved an adequate level of accuracy on average. However, local misadaptations, e.g., at the vertebra rib articulation, cause significant local maximal errors. In one case, most parts of the model adapted to neighboring structures caused by an imprecise object detection. In order to give a visual impression, Fig. 4 (a) shows the segmentation result in one image slice of one arbitrarily chosen data set and the corresponding adapted model in Fig. 4 (b).

Compared to the positioning and adaptation of the complete model, we obtained similar results for the segmentation of the vertebral column using the iterative adaption from Sect. 2.3. In each case, we chose the lowest vertebra shown in the data set as start vertebra. The mean values for $d_{\text {mean }}$ and $d_{\text {max }}$ were 1.12 and $5.56 \mathrm{~mm}$. However, in one data set the approach completely failed. Due to a vertebra fracture, one vertebra could not be segmented correctly, so that the positioning of all subsequent models was incorrect resulting in misadaptation.



(a) Image Slice

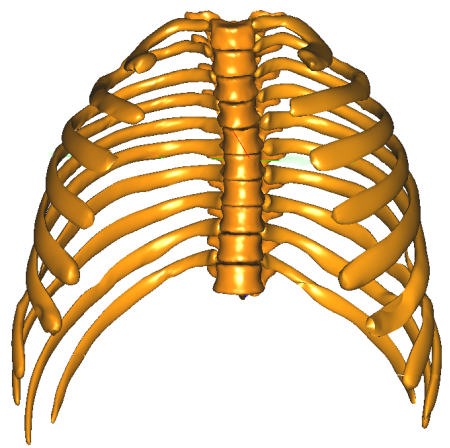

(b) Adapted Model

Fig. 4. Adapted mean model to unseen image data. Figure (a) shows the result in one image slice. Color coding illustrates labeling (see electronic version). Each label corresponds to the correct anatomical object. The arrow points to a local misadaptation. The adapted model is shown in (b).

\section{Conclusion}

Model-based segmentation requires careful positioning. Especially, in the case of the rib cage with its amount of similar neighboring structures, misadaptation to neighboring structures is a crucial point. However, with the developed 
positioning, the individual models adapted in almost all cases correctly to their corresponding image objects. In 16 out of 18 data sets, detection, labeling, and segmentation succeeded with a mean distance between the true and the detected object surface of $1.27 \mathrm{~mm}$ in case of the vertebrae and $0.36 \mathrm{~mm}$ in case of the ribs. An alternative approach for spine segmentation was performed that uses object relations derived from the mean model. This approach was successful in 17 out of 18 cases with a mean distance of $1.12 \mathrm{~mm}$. We believe that we achieved an acceptable level of accuracy for some applications. If a higher accuracy is needed, the results may serve as a good basis for a locally detailed delineation.

\section{References}

1. Shen, H., Liang, L., Shao, M., Qing, S.: Tracing Based Segmentation for the Labeling of Individual Rib Structures in Chest CT Volume Data. In: Barillot, C., Haynor, D.R., Hellier, P. (eds.) MICCAI 2004. LNCS, vol. 3217, pp. 967-974. Springer, Heidelberg (2004)

2. Staal, J., van Ginneken, B., Viergever, M.A.: Automatic rib segmentation and labeling in computed tomography scans using a general framework for detection, recognition and segmentation of objects in volumetric data. Medical Image Analysis 11, 35-46 (2006)

3. Lorenz, C., von Berg, J.: A comprehensive shape model of the heart. Medical Image Analysis 10, 657-670 (2006)

4. Weese, J., Kaus, M., Lorenz, C., Lobregt, S., Truyen, R., Pekar, V.: Shape constrained deformable models for $3 \mathrm{~d}$ medical image segmentation. In: Insana, M.F., Leahy, R.M. (eds.) IPMI 2001. LNCS, vol. 2082, pp. 380-387. Springer, Heidelberg (2001)

5. Fitzgibbon, A.W., Pilu, M., Fisher, R.B.: Direct Least Squares Fitting of Ellipses. IEEE Transaction on Pattern Anaylysis and Machine Intelligence 21(5), 476-480 (1996)

6. Lorenz, C., von Berg, J.: Fast automated object detection by recursive casting search rays. Computer Assisted Radiology and Surgery, 230-235 (2005)

7. Besl, P.J.: A Method For Registration of 3-D Shapes. IEEE Transaction on Pattern Analysis and Machine Intelligence 14(2), 239-256 (1992)

8. Donato, G., Belongie, S.: Approximate Thin Plate Spline Mappings. In: Heyden, A., Sparr, G., Nielsen, M., Johansen, P. (eds.) ECCV 2002. LNCS, vol. 2352, pp. 21-31. Springer, Heidelberg (2002) 